\title{
Recombination analysis of Soybean mosaic virus sequences reveals evidence of RNA recombination between distinct pathotypes
}

\author{
Alla G Gagarinova ${ }^{1,2,4}$, Mohan Babu ${ }^{1}$, Martina V Strömvik ${ }^{3}$ and \\ Aiming Wang*1,2
}

\begin{abstract}
Address: ${ }^{1}$ Southern Crop Protection and Food Research Centre, Agriculture and Agri-Food Canada, 1391 Sandford St., London, Ontario, N5V 4T3, Canada, ${ }^{2}$ Department of Biology, The University of Western Ontario, Biological \& Geological Building, 1151 Richmond St., London, Ontario, N6A 5B7, Canada, ${ }^{3}$ Department of Plant Science, McGill University, 21111 Lakeshore Rd., Ste. Anne de Bellevue, Québec, H9X 3V9, Canada and ${ }^{4}$ Department of Molecular Genetics, The University of Toronto, Toronto, M5S 1A8, Canada
\end{abstract}

Email: Alla G Gagarinova - alla.gagarinova@utoronto.ca; Mohan Babu - mohanbabu_r@yahoo.com; Martina V Strömvik - martina.stromvik@mcgill.ca; Aiming Wang* - wanga@agr.gc.ca

* Corresponding author

Published: 26 November 2008

Virology Journal 2008, 5:143 doi:10.1186/1743-422X-5-143

This article is available from: http://www.virologyj.com/content/5/I/l43

(c) 2008 Gagarinova et al; licensee BioMed Central Ltd.

This is an Open Access article distributed under the terms of the Creative Commons Attribution License (http://creativecommons.org/licenses/by/2.0), which permits unrestricted use, distribution, and reproduction in any medium, provided the original work is properly cited.
Received: 3 August 2008

Accepted: 26 November 2008

\begin{abstract}
RNA recombination is one of the two major factors that create RNA genome variability. Assessing its incidence in plant RNA viruses helps understand the formation of new isolates and evaluate the effectiveness of crop protection strategies. To search for recombination in Soybean mosaic virus (SMV), the causal agent of a worldwide seed-borne, aphid-transmitted viral soybean disease, we obtained all full-length genome sequences of SMV as well as partial sequences encoding the $\mathrm{N}$ terminal most (PI protease) and the C-terminal most (capsid protein; $\mathrm{CP}$ ) viral protein. The sequences were analyzed for possible recombination events using a variety of automatic and manual recombination detection and verification approaches. Automatic scanning identified 3, 10, and 17 recombination sites in the $\mathrm{PI}, \mathrm{CP}$, and full-length sequences, respectively. Manual analyses confirmed 10 recombination sites in three full-length SMV sequences. To our knowledge, this is the first report of recombination between distinct SMV pathotypes. These data imply that different SMV pathotypes can simultaneously infect a host cell and exchange genetic materials through recombination. The high incidence of SMV recombination suggests that recombination plays an important role in SMV evolution. Obtaining additional full-length sequences will help elucidate this role.
\end{abstract}

\section{Findings}

Soybean mosaic virus (SMV) is a member of the genus Potyvirus, the family Potyviridae. It is one of the most devastating viral pathogens of soybean crops causing severe symptoms such as mosaic, mottling, chlorosis and rugosity in leaves, as well as reductions in plant growth with yield losses of up to $100 \%$ [1-4]. Like all potyviral genomes, the SMV genome is a single-stranded, positivesense RNA molecule that is approximately $10 \mathrm{~kb}$ in length and contains a single open reading frame [4-6]. It encodes a large polyprotein that is co- and post-translationally cleaved into 11 final protein products [4-6]. SMV is found in all soybean-growing regions of the world. In the United States, at least $98 \mathrm{SMV}$ isolates have been documented $[7,8]$. Based on their differential interactions with SMV resistant cultivars, these isolates are classified into seven distinct strain groups, G1 through G7 [7,8]. Similarly, five (A to E) and eight (Sa to Sh) SMV strains have been 
reported in Japan and China, respectively [9-11]. Though the pathotypic relationships between the SMV groups in the United States and the strains in China and Japan are not clear, these data clearly suggest a high genetic diversity of SMV.

The two major factors that contribute to the variability and evolution of RNA viruses are mutation introduced by the viral RNA-dependent RNA polymerase and recombination between different viral RNA molecules [12]. The mutation rate varies between virus species, and the recombination frequency is dependent on the degree of sequence similarity between the sequences involved, the length of viral genome and the presence of recombination hot spots [12-14]. Mutation has been demonstrated to be responsible for the emergence of new SMV isolates that differentiate from their parental isolates by breaking resistance in soybean under both field and laboratory conditions [15-17]. However, the role of RNA recombination in SMV evolution still remains unknown.

We evaluated RNA recombination in SMV. All partial SMV sequences encoding the N-terminal most (P1 protease) and the C-terminal most (capsid protein; $\mathrm{CP}$ ) as well as full-length SMV sequences were retrieved from GenBank [Additional file 1]. The alignments were performed using ClustalW [18]. Subsequently, the automatic recombination scans of the sequence alignments were performed using Recombination Detection Program v.3.31 (RDP3) with default settings [19]. RDP3 scans all possible triplet combinations of sequences to identify and statistically test the recombination signals [19]. When two (parental) sequences are joined to form a recombinant (daughter) sequence, recombination signals may be detected in the parental, daughter, any descendant, and other closely related isolates. Thus, it is possible that a single recombination event can be counted several times. RDP3 overcomes this complication by automatically combining recombination signals to identify a minimum set of unique recombination events that account for the observed similarity patterns among sequences.

Our RDP3 analyses identified many unique recombination events in full-length, P1, and CP alignments (Figure 1) [Additional file 2]. However, because of the apparently large number of ancestral and overlapping recombination signals in full-length SMV sequences, final assignments of parental and daughter designations in the identified unique recombination events were affected by the order in which the sequences were analyzed. This ambiguity was likely caused by the limited number of full-length genome sequences for many SMV strains. Manual examination of the RDP3 results did not reveal a better set of the unique recombination events, suggesting the complex similarity patterns among SMV sequences could arise through recombination in diverse ways (data not shown). In accordance with the parsimony principle, we have presented the output that explains the relationships between SMV sequences in all alignments by the smallest number of recombination events (Figure 1B) [Additional file 2].

We further examined more recent, in evolutionary terms, recombination events in full-length SMV sequences. Putative non-recombinant and recombinant sequences were identified using Simplot [20] with 200 bp window and 20 bp step sizes. Location and significance of each putative recombination site was tested using the $\chi^{2}$ test, or the informative sites analysis, implemented in Simplot [2123 ]. The recombination site was placed where the highest significant $\chi^{2}$ value was obtained. Two blocks of sequences, on either side of the recombination site, each from a single parent, were compared to assess the likelihood of recombination at the given site. First, non-SMV potyvirus and, subsequently, a distantly related isolate of SMV were used as outgroups in $\chi^{2}$ tests to increase the number of informative sites and to narrow down the location of the recombination. Essentially, locations of the putative recombination sites identified by the Simplot coincided with the locations of the unique recombination events identified by RDP3 (data not shown). Recombination sites in $\mathrm{CN} 18, \mathrm{HZ}$, and $\mathrm{HH} 5$ sequences were supported with a $P$-value $<0.05$ by the $\chi^{2}$ test. However, no recombination sites were manually assigned to these isolates since a large number of un-uniformly distributed informative sites supported grouping of these isolates with the outgroup in all tests, indicating that isolates $\mathrm{CN} 18, \mathrm{HZ}$, and HH5 were too diverged from all other SMV sequences for the $\chi^{2}$ test (data not shown). Nevertheless, a number of significant recombination events were identified in G5, G7H, and G7f sequences.

Two SMV isolates, G5 and G7H, though belonging to two different pathotypes, were previously reported to be closely related to each other based on full-length genome sequence comparison [24]. Consistently with this finding, isolate G7H indeed clustered with isolate G5 and not with pathotype G7 isolates in a phylogenetic tree constructed from the full-length SMV genome sequences (Figure 1A). Recombination sites, ' $w$ ', ' $x$ ' and ' $z$ ' in G5 and ' $w$ ', ' $y$ ', and ' $\mathrm{z}$ ' in G7H were supported statistically with a $P$-value < 0.05 by the $\chi^{2}$ test. Following were the locations of these sites in respect to the G5 and G7H sequences: ' $w$ ' 4199 4208, 'x' 5441 - 5546, 'y' 5546 - 5603, and 'z' 6362 6410. Similarity patterns and phylogenetic trees constructed for the sequence alignment regions demarcated by the recombination sites confirmed two recombination events in each of the isolates: ' $w$ ' and ' $x$ ' in G5 and ' $y$ ' and ' $z$ ' in G7H (Figure 2) [Additional file 3] [Additional file 4]. These recombination sites in G5 and G7H were supported statistically with a $P$-value $<0.001$ [Additional file 3] 
A

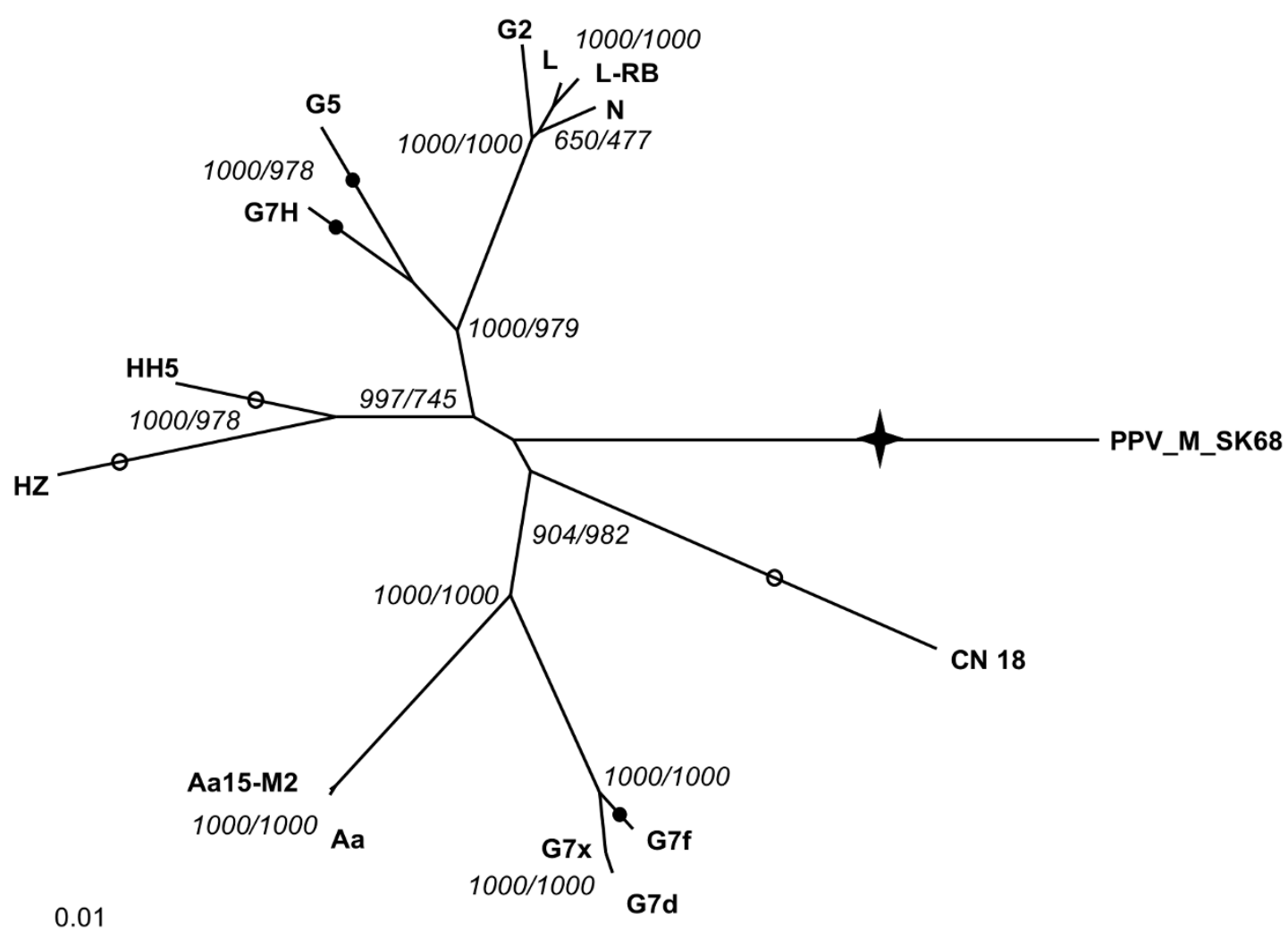

B

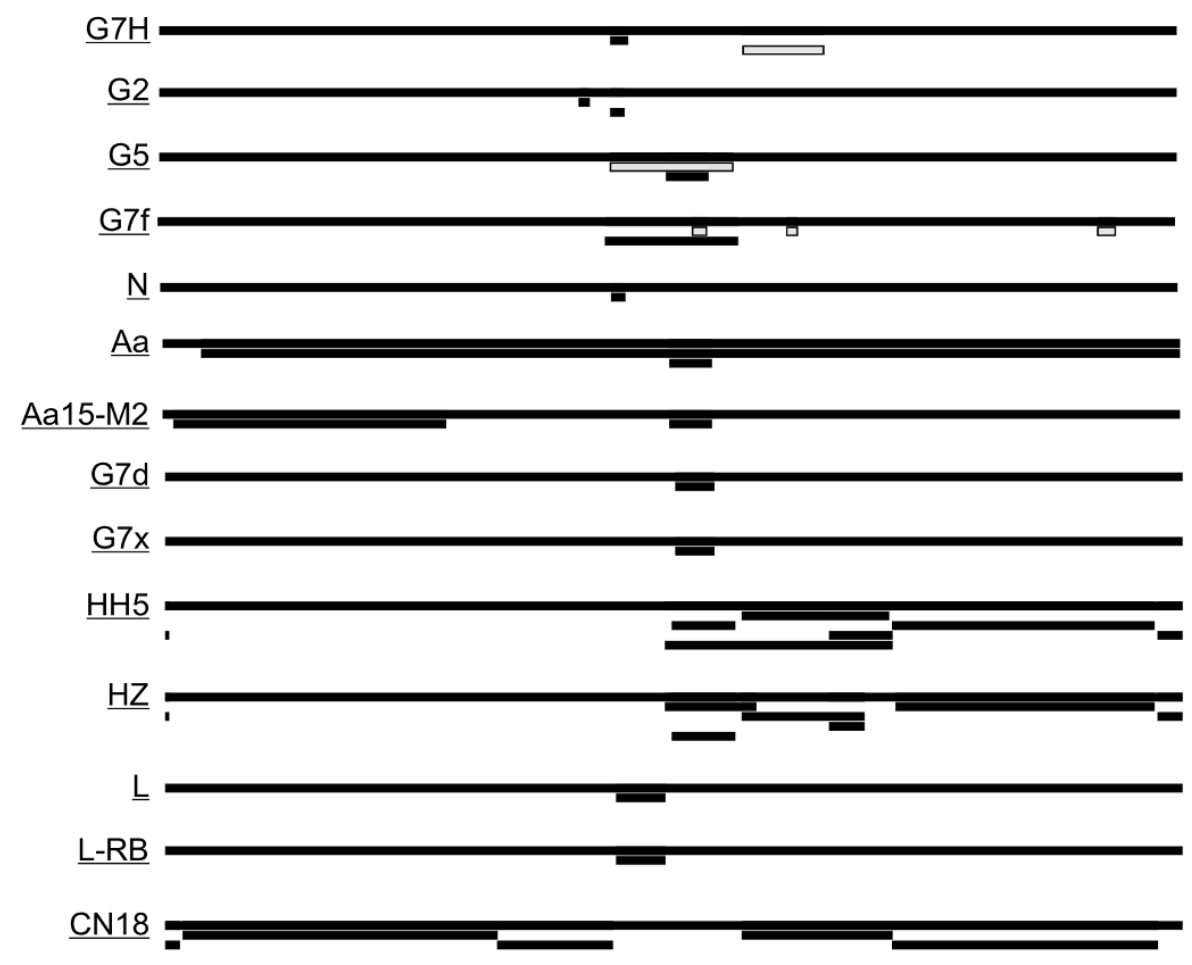

Figure I (see legend on next page) 
Figure I (see previous page)

Recombination in full-length SMV sequences. A. Phylogenetic relationships of SMV isolates to each other and to PPV as an outgroup. Phylogenetic tree was constructed using full-length nucleotide sequences of isolates L [GenBank: EU87|724], LRB [GenBank: EU87I725], G2 [GenBank: S42280.I], N [GenBank: D00507.2], Aa [GenBank: ABI00442.I], Aa I 5-M2 [GenBank: ABI00443.I], G5 [GenBank: AY294044.I], G7H [GenBank: AY294045.I], G7d [GenBank: AY2I6987.I], G7 referred as G7x [GenBank: AY2I60I0.I], and G7 referred as G7f [GenBank: AF24I739.I], CNI8 [GenBank: A]619757], HH5 [GenBank: AJ310200], HZ [GenBank: AJ312439], as well as PPV [GenBank: M92280.I], as the outgroup, and the Neighbour Joining function of ClustalX [34]. Topologies of the Bayesian [35] as well as the 1000 times bootstrapped least squares [36] and maximum likelihood [37] phylogenetic trees were same (data not shown). Bootstrap values for the Neighbour Joining and the maximum likelihood phylogenetic trees, out of 1000 replicates, are given at the nodes before and after the slanted line, respectively. For presentation purposes, the line marked with a star was shortened from 0.35 I 45 to 0.04I45. Automated RDP3 recombination analysis identified recombination events in all SMV isolates [please also see Additional file I]. Filled circles demarcate likely times, when in evolution of SMV manually verified recombination events took place, while empty circles demarcate significant $(P$-value $<0.05)$ recombination events where the likely recombinant isolates were determined to be too far diverged from all available SMV sequences for the $\chi^{2}$ analysis of recombination and thus recombination analyses results were considered inconclusive. B. Locations of unique recombination events identified by RDP3, in relation to the full-length sequence alignment [please also see the Additional file I]. Each full-length genome is represented by a long black bar and the corresponding underlined isolate name, given to the left of the bar. The figure shows a total of 17 unique recombination events, demarcated by the bars below the genomes the recombinant fragments have been integrated into. When an ancestral unique recombination event can be found in more than one daughter sequence, the recombination event is displayed with all corresponding daughter sequences. Locations of the unique recombination events identified by RDP, corresponding to the manually verified recombination sites, are shown with grey bars [please also see Additional file I].

[Additional file 4]. These phylogenetic and recombination analysis results suggest that majority of G5 and G7H genome sequences were derived from a common ancestor more closely related to the G2 group of isolates, while fragments between recombination sites ' $w$ ' and ' $x$ ' in G5 and ' $y$ ' and ' $z$ ' in G7H are more closely related to G7x and G7d (Figure 2). The high-confidence phylogenetic grouping of G7H with G7d and G7x is consistent with the location of factors distinguishing G7 pathotype from G2 and G5 pathotypes somewhere between recombination sites ' $y$ ' and ' $z$ ', in the genome region encoding the C-terminal part of $6 \mathrm{~K} 2-\mathrm{VPg}$ and the N-terminal part of NIa-Pro.

Analyses of the G7f genome revealed six recombination events, 'a' through ' $\mathrm{f}$ ', that occurred in the formation of this isolate. G7f was most similar to G7x and G7d isolates along most of its genome, but was more similar to G2 between recombination sites 'a' and 'b', ' $c$ ' and ' $d$ ', 'e' and ' $f$ ' [Additional file 5]. The following were the nucleotide position numbers of recombination event locations in respect to the G7f sequence: 'a' 5102-5114, 'b' 52525285, 'c' 6021-6026, 'd' 6140-6176, 'e' 8846-8858, 'f' 9008-9035. In $\chi^{2}$ tests, all 6 recombination sites were supported with $P$-value $<0.0025$. However, either mutation, coupled with strong selection, or recombination could result in an isolate being most similar to two different isolates in the neighbouring regions [25]. Selection of mutations would be expected to act at the amino acid sequence level as, to the best of our knowledge, avirulence determinants have only been reported to act at this level [26-32]. On the other hand, recombination may or may not affect the amino acid sequence of the resulting chimera. In this study, most sites that supported grouping of G7f with G2 were silent [Additional file 6] [Additional file 7], providing support for recombination rather than selection hypotheses to explain these similarities. All manually identified G5, G7H, and G7f recombination sites were recapitulated by manual GENECONV test implemented in RDP3 (data not shown).

The manually generated and verified results presented here provide the strong evidence of recombination in SMV. The most parsimonious output of RDP3 for fulllength sequences [Additional file 2] partially, but better than other RDP3 outputs, coincided with the manual sequence comparisons results, emphasizing the need for the manual verification of automatically generated results. Furthermore, the G5 and G7H recombination analysis results suggest recombination analysis as a tool for directing experiments to identify avirulence determinants and develop novel crop protection strategies. However, utility of recombination detection in SMV is still limited by the lack of representative full-length genome sequences for most of SMV strains. Availability of representative sequences with associated pathogenicity profiles will allow elucidating the evolutionary history of SMV and deriving testable hypotheses about SMV-soybean interactions.

In spite of limitations to analyzing SMV recombination, application of conceptually different, complementary approaches allowed us to detect recombination sites previously missed by Chare and Holmes [33]. Our work showed, for the first time, that recombination occurred 


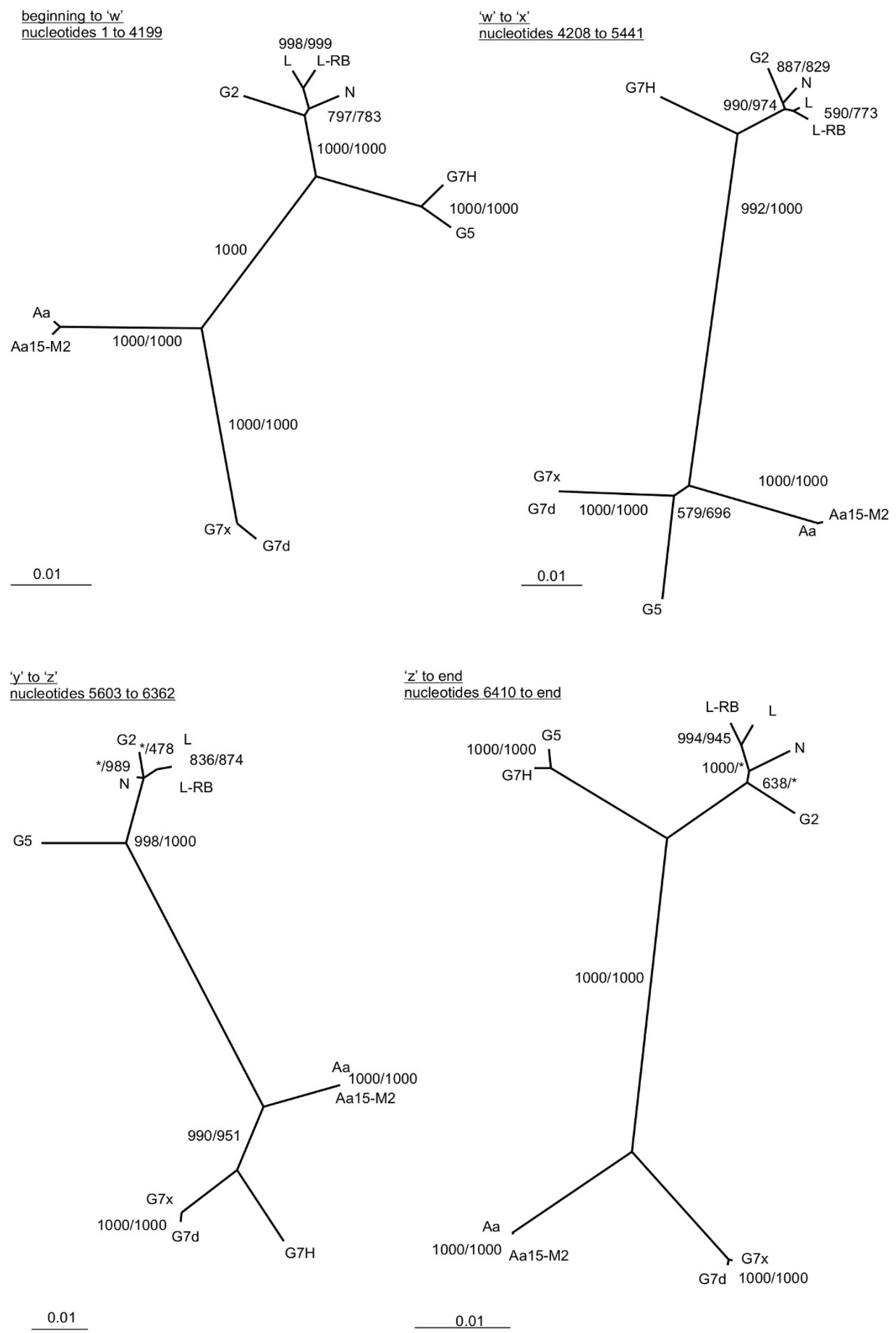

Figure 2 (see legend on next page) 
Figure 2 (see previous page)

Phylogenetic trees for the alignment regions demarcated by $\mathbf{G 5}$ and $\mathbf{G 7 H}$ recombination sites. Non-recombinant, as determined by the manual recombination analysis (see manuscript text), as well as G5 and G7H sequences were included in the phylogenetic tree construction. The designations for the fragments are given at the top, to the left of each tree. Bayesian [35] as well as bootstrapped Neighbour Joining [34], least squares [36], and maximum likelihood [37] trees were constructed for each region. Topologies of the trees generated by the four methods for the same region were same, with exception of how $C 2$ and $N$ sequences related to each other and to $L$ and $L-R B$ isolates from recombination site ' $y$ ' to the end of the alignment. Shimodaira-Hasegawa (SH) test [38] was used to select the best of the competing but very similar topologies for each sequence region (date not shown). The tree topology that obtained the highest SH score of I is presented for each region. Bootstrap values out of 1000 replicates, produced by the Neighbour Joining and the maximum likelihood methods are given at the nodes, before and after the slanted line, respectively. A star given instead of the number indicates that the respective method did not agree with the topology of the optimal tree identified by the SH test at that particular node. Topologies of all trees were tested against each other and the topologies of the trees presented here were found optimal (SH score: I). SH scores of 0 were obtained when topologies of the trees from between recombination sites were tested against sequence alignments for the regions on the basis of which the given tree was not generated. The same SH score of 0 was obtained when the tree topologies for the regions from the beginning of the sequence alignment to ' $w$ ' and from ' $z$ ' to end of the sequence alignment were tested against sequence alignments between the recombination sites. Collectively, these results indicated that the different topologies cannot substitute for each other in explaining the variability of SMV sequences between G5 and G7H recombination sites that we identified.

during SMV evolution among distinct viral isolates and thus provided evidence that at least two distinct viral SMV pathotypes can simultaneously infect a host cell and exchange genetic materials through RNA recombination. The high frequency of recombination detected in SMV suggests that recombination plays an important role in SMV evolution and this should be considered when novel antiviral strategies are developed.

\section{Competing interests}

The authors declare that they have no competing interests.

\section{Authors' contributions}

AGG acquired SMV genomic sequences and performed the analysis. AGG, MB, MVS, and AW interpreted the data. AW conceived the study. AGG and AW wrote the paper. All authors critically reviewed and approved the final manuscript.

\section{Additional material}

\section{Additional File 1}

List of full-length and partial $(P 1, C P)$ sequences of SMV analysed for recombination. A list of all sequences and the corresponding Genbank accession numbers are provided.

Click here for file

[http://www.biomedcentral.com/content/supplementary/1743422X-5-143-S1.pdf]

\section{Additional File 2}

Summary of unique recombination events identified by the Recombination Detection Program v.3.31 (RDP3). Our RDP3 automated analyses using RDP, GENECONV, Bootscan, MaxChi, Chimera, and SiScan methods [19] identified many highly significant recombination signals in full-length, P1, and CP alignments [please see Additional file 1 for the list of accession numbers for all analyzed sequences]. However, when two (parental) sequences are joined to form a recombinant (daughter) sequence, recombination signals will be detected in all descendants of the parental and daughter isolates as well as related sequences, provided the recombination signals have not been obscured by subsequent recombination events or strong selection. All detected recombination signals were automatically combined by RDP3 into sets of unique recombination events. The final set of the unique recombination events depended on the order in which the sequences were analyzed. This effect of sequence analysis order on the generated set of unique recombination events was particularly strong for the full-length sequences, where a large number of ancestral and overlapping recombination signals were found. This ambiguity was likely increased by the lack of full-length genome sequences representing many of the SMV strains. Manual investigation of the RDP3 results did not suggest that any one set of the unique recombination events was better than another: the complex similarity patterns between SMV sequences could arise through recombination in a number of ways (data not shown). Therefore, in accordance with the parsimony principle, we presented the output that explains the relationships between SMV isolates by the smallest number of recombination events. The largest number of unique recombination events was consistently detected by RDP3 in fulllength sequences despite the fact that the smallest number of these sequences was analyzed. This may have to do with the fact that complete evolutionary history is preserved in full-length sequences, but not the partial sequences such as $P 1$ and CP that were also analyzed here. More fulllength SMV sequences must be obtained in order to describe the broad picture of how recombination affected evolution of SMV. Obtaining additional sequences will also aid in resolving uncertainties about parental and daughter isolate identities and narrowing down the locations of undetermined break points (recombination sites).

Click here for file

[http://www.biomedcentral.com/content/supplementary/1743422X-5-143-S2.pdf] 


\section{Additional File 3}

Supplemental Figure 1. Similarity plots with G5 as the query isolate Lists of isolates included in the analyses with their corresponding line colors are shown in the legend box. Locations of sites ' $w$ ', ' $x$ ', ' $y$ ', and ' $z$ ' are demarcated with vertical lines and the green underlined letters. Regions used for "find sites" analyses are marked with rectangles; names for the query, first and second parental, as well as outgroup isolates, with respective numbers of informative sites, supporting each grouping, and the $\chi^{2}$ values are given for each recombination site in matching colors. Click here for file [http://www.biomedcentral.com/content/supplementary/1743422X-5-143-S3.pdf]

\section{Additional File 4}

Supplemental Figure 2. Similarity plots with $\mathrm{G} 7 \mathrm{H}$ as the query isolate. Lists of isolates included in the analyses with their corresponding line colors are shown in the legend box. Locations of sites ' $w$ ', ' $x$ ', ' $y$ ', and ' $z$ ' are demarcated with vertical lines and the green underlined letters. Regions used for "find sites" analyses are marked with rectangles; names for the query, first and second parental, as well as outgroup isolates, with respective numbers of informative sites, supporting each grouping, and the $\chi^{2}$ values are given for each recombination site in matching colors. Click here for file [http://www.biomedcentral.com/content/supplementary/1743422X-5-143-S4.pdf]

\section{Additional File 5}

Supplemental Figure 3. Similarity plot with G7f as the query isolate. List of isolates included in the analysis with their corresponding line colors are shown in the legend box. Location of each statistically significant recombination site is demarcated with a vertical line and a green underlined letter, adjacent to the line. Names for the query, first and second parental isolates, and outgroups with corresponding numbers of informative sites, supporting each grouping are given in red and blue, respectively. The $\chi^{2}$ values for each recombination site are given in italicized underlined font, adjacent to the vertical line demarcating each respective recombination site.

Click here for file

[http://www.biomedcentral.com/content/supplementary/1743422X-5-143-S5.pdf]

\section{Additional File 6}

Supplemental Table 1. Effect of informative site nucleic acid differences on amino acid composition in analyses of G7f recombination events with remotely related non-SMV potyvirus sequence (PPV) as outgroup. Informative sites that are also found in analyses with Aa as outgroup [see Additional file 7] are given in italics.

Click here for file

[http://www.biomedcentral.com/content/supplementary/1743422X-5-143-S6.pdf]

\section{Additional File 7}

Supplemental Table 2. Effect of informative site nucleic acid differences on amino acid composition in analyses of G7f recombination events with Aa as outgroup. Informative sites that are also found in analyses with nonSMV potyvirus sequence (PPV) as outgroup [see Additional file 6] are given in italics.

Click here for file

[http://www.biomedcentral.com/content/supplementary/1743422X-5-143-S7.pdf]

\section{Acknowledgements}

This work was supported by Ontario Soybean Growers, the AAFC Crop Genomics Initiative and the Natural Sciences and Engineering Research Council of Canada. AGG was a recipient of Ontario Graduate Scholarship and Western Graduate Research Scholarship.

\section{References}

I. Arif M, Hassan S: Evaluation of resistance in soybean germplasm to Soybean mosaic potyvirus under field conditions. J Biol Sci 2002, 2:60I-604.

2. Gunduz I, Buss GR, Chen P, Tolin SA: Genetic and phenotypic analysis of Soybean mosaic virus resistance in PI 88788 soybean. Phytopathology 2004, 94:687-692.

3. Liao L, Chen P, Buss GR, Yang Q, Tolin SA: Inheritance and allelism of resistance to Soybean mosaic virus in Zaol 8 soybean from China. J Hered 2002, 93:447-452.

4. Babu M, Gagarinova AG, Brandle JE, Wang A: Association of the transcriptional response of soybean plants with soybean mosaic virus systemic infection. J Gen Virol 2008, 89: I069- 1080.

5. Urcuqui-Inchima S, Haenni AL, Bernardi F: Potyvirus proteins: a wealth of functions. Virus Res 200I, 74:157-175.

6. Chung BY-W, Miller WA, Atkins JF, Firth AE: An overlapping essential gene in the Potyviridae. Proc Natl Acad Sci USA 2008, 105:5897-5902.

7. Cho EK, Goodman RM: Strains of soybean mosaic virus classification based on virulence in resistanct soybean cultivars. Phytopathology 1979, 69:467-470.

8. Cho EK, Goodman RM: Evaluation of resistance in soybeans to soybean mosaic virus strains. Crop Sci 1982, 22: I I33-I I 36.

9. Takahashi K, Tanaka T, lida W, Tsuda Y: Studies on virus diseases and causal viruses of soybean in Japan. Tohoku Natl Agric Exp Stn Bull 1980, 62: I-130.

10. Chen YX, Xue BD, Hu YZ, Fang ZD: Identification of two new starins of soybean mosaic virus. Acta Phytophyl Sin 1986, | 3:22|-226.

II. Pu ZQ, Cao Q, Fang DC, Xi BD, Fang CT: Identification of strains of soybean mosaic virus. Acta Phytophyl Sin 1982, 9:15-20.

12. García-Arenal F, Fraile A, Malpica JM: Variation and evolution of plant virus populations. Int Microbiol 2003, 6:225-232.

13. Lai MM: RNA recombination in animal and plant viruses. Microbiol Rev 1992, 56:61-79.

14. Gallei $A$, Pankraz A, Thiel HJ, Becher P: RNA recombination in vivo in the absence of viral replication. I Virol 2004, 78:627|-628I.

15. Choi BK, Koo JM, Ahn HJ, Yum HJ, Choi CW, Ryu KH, Chen P, Tolin SA: Emergence of Rsv-resistance breaking Soybean mosaic virus isolates from Korean soybean cultivars. Virus Res 2005, I I 2:42-5I.

16. Gagarinova AG, Babu M, Poysa V, Hill JH, Wang A: Identification and molecular characterization of two naturally occurring Soybean mosaic virus isolates that are closely related but differ in their ability to overcome Rsv4 resistance. Virus Res 2008 in press.

17. Hajimorad MR, Eggenberger AL, Hill JH: Evolution of Soybean mosaic virus-G7 molecularly cloned genome in Rsv I-genotype soybean results in emergence of a mutant capable of evading Rsv I-mediated recognition. Virology 2003, 3 I 4:497-509.

18. Thompson JD, Higgins DG, Gibson TJ: CLUSTAL W: improving the sensitivity of progressive multiple sequence alignment through sequence weighting, position-specific gap penalties and weight matrix choice. Nucleic Acids Res 1994, 22:4673-4680.

19. Martin DP, Williamson C, Posada D: RDP2: recombination detection and analysis from sequence alignments. Bioinformatics 2005, $21: 260-262$.

20. Lole KS, Bollinger RC, Paranjape RS, Gadkari D, Kulkarni SS, Novak NG, Ingersoll R, Sheppard HW, Ray SC: Full-length human immunodeficiency virus type I genomes from subtype C-infected seroconverters in India, with evidence of inter subtype recombination. J Virol 1999, 73:152-160.

21. Robertson DL, Hahn BH, Sharp PM: Recombination in AIDS viruses. I Mol Evol 1995, 40:249-259.

22. Robertson DL, Sharp PM, McCutchan FE, Hahn BH: Recombination in HIV-I. Nature 1995, 374:124-126.

23. Smith JM: Analyzing the mosaic structure of genes. J Mol Evol 1992, 34:126-129. 
24. Lim WS, Kim YH, Kim KH: Complete genome sequences of the genomic RNA of Soybean mosaic virus strains G7H and G5. Plant Pathol 2003, 19:17I-176.

25. Domingo E, Holland J]: RNAvirus mutations and fitness for survival. Annu Rev Microbiol 1997, 5 I: I5 I-78.

26. Hajimorad MR, Eggenberger AL, Hill JH: Loss and gain of elicitor function of Soybean mosaic virus G7 provoking Rsv I-mediated lethal systemic hypersensitive response maps to P3. J Virol 2005, 79: I2I $15-1222$.

27. Rajamaki ML, Valkonen JPT: The $6 \mathrm{~K} 2$ protein and the VPg of potato virus $A$ are determinants of systemic infection in Nicandra physaloides. Mol Plant-Microbe Interact 1999, 1 2:1074-1081.

28. Jenner CE, Sanchez F, Nettleship SB, Foster GD, Ponz F, Walsh JA: The cylindrical inclusion gene of Turnip mosaic virus encodes a pathogenic determinant to the Brassicaresistance gene TuRBO I. Mol Plant-Microbe Interact 2000, 13:1 102-I 108

29. Kühne T, Shi N, Proeseler G, Adams MJ, Kanyuka K: The ability of a bymovirus to overcome the rym4-mediated resistance in barley correlates with a codon change in the VPg coding region on RNAI. J General Virol 2003, 84:2853-2859.

30. Ruffel S, Gallois JL, Lesage ML, Caranta C: The recessive potyvirus resistance gene pot- $I$ is the tomato orthologue of the pepper pvr2-elF4E gene. Mol Gen Genomics 2005, 274:346-353.

31. Hjulsager CK, Olsen BS, Kjaer Jensen DM, Cordea MI, Krath BN, Johansen IE, Lund OS: Multiple determinants in the coding region of Pea seed-borne mosaic virus $P 3$ are involved in virulence against sbm-2 resistance. Virology 2006, 355:52-6I.

32. Desbiez C, Gal-On A, Girard M, Wipf-Scheibel C, Lecoq H: Increase in Zucchini yellow mosaic virus symptom severity in tolerant zucchini cultivars is related to a point mutation in $\mathbf{P} 3$ protein and is associated with a loss of relative fitness on susceptible plants. Phytopathology 2003, 93:| 1478-1484.

33. Chare ER, Holmes EC: A phylogenetic survey of recombination frequency in plant RNA viruses. Arch Virol 2006, I 5 I:933-946.

34. Thompson JD, Gibson TJ, Plewniak F, Jeanmougin F, Higgins DG: The ClustalX windows interface: flexible strategies for multiple sequence alignment aided by quality analysis tools. Nucleic Acids Res 1997, 24:4876-4882.

35. Ronquist F, Huelsenbeck JP: MRBAYES 3:Bayesian phylogenetic inference under mixed models. Bioinformatics 2003, 19:1572-1574

36. Felsenstein J: PHYLIP - Phylogeny Inference Package (Version 3.2). Cladistics 1989, 5:164-166.

37. Guindon S, Gascuel O: A simple, fast, and accurate algorithm to estimate large phylogenies by maximum likelihood. Syst Biol 2003, 52:696-704.

38. Shimodaira H, Hasegawa M: Multiple comparisons of log-likelihoods with applications to phylogenetic inference. Mol Biol Evol 1999, 16:1114-1116.
Publish with Bio Med Central and every scientist can read your work free of charge

"BioMed Central will be the most significant development for disseminating the results of biomedical research in our lifetime. "

Sir Paul Nurse, Cancer Research UK

Your research papers will be:

- available free of charge to the entire biomedical community

- peer reviewed and published immediately upon acceptance

- cited in PubMed and archived on PubMed Central

- yours - you keep the copyright
BioMedcentral 